\title{
Avances en el desarrollo de un Repositorio para Recursos Educativos Abiertos
}

\author{
Silvia Vanesa Torres, María Soledad Zangla, Marcela Cristina Chiarani \\ UNSL, Ejercito de los Andes 950 \\ San Luis, Capital \\ \{svtorres, szangla, mchiarani\}@unsl.edu.ar
}

\begin{abstract}
Resumen. En este artículo se presenta el avance en el desarrollo de un repositorio de recursos educativos abiertos, creado con el objetivo de compartir experiencias y materiales educativos. En la actualidad uno de los enfoques, ampliamente aceptado, en la aplicación de las TIC en educación se basa en un nuevo paradigma de diseño de materiales de aprendizaje, poniendo énfasis en la reutilización de contenidos. Por consecuencia, surge la necesidad de almacenar, buscar, recuperar, consultar y descargar estos materiales de aprendizaje. Desde el 2009, el Centro de Informática Educativa (CIE), lleva a cabo la producción de Materiales Educativos Digitales (MED), a partir de una perspectiva interdisciplinaria con docentes de diferentes niveles educativos de la provincia de San Luis. El principal propósito es lograr que los materiales sean utilizados en las aulas. Considerando la idea de código abierto y el movimiento de acceso libre al conocimiento, desde el proyecto de investigación, PROICO N³0212 "Herramientas Informáticas Avanzadas para Gestión de Contenido digitales para Educación" de la Facultad de Ciencias Físico Matemáticas y Naturales de la Universidad Nacional de San Luis, se tiene como objetivo investigar, modificar y desarrollar herramientas de software libre que permitan crear MED. Se busca con ello propiciar un marco de colaboración para el desarrollo de recursos educativos, a modo de potenciar la generación y reusabilidad de contenidos digitales.
\end{abstract}

Palabras Claves: MED, Repositorio, código abierto

\begin{abstract}
This paper presents the progress in the development of a repository of open educational resources, created with the aim of sharing experiences and educational materials. At current approach, widely accepted in the application of ICT in education is based on a new design paradigm for learning materials , emphasizing the reuse of content . Consequently, the need to store, search, retrieve, view, and download these learning materials. Since 2009, the Center for Computing Education ( ICE) conducts the production of Digital Materials Education (MED ), from an interdisciplinary perspective with teachers from different educational levels in the province of San Luis . A main purpose is to use the materials in classrooms. Taking in mind the idea of open source movement and free access to knowledge , the research project No. 30212 PROICO "Advanced Computer Tools for Managing Digital Content for Education " , Faculty of Physics and Mathematics, and the National University San Luis, the objective is to research, develop and modify free software tools that create MED. With the intention of fostering a collaborative framework for the development of educational resources to enhance the generation mode and reusability of digital contents.
\end{abstract}

Keywords: MED, Repositorio, open source, 


\section{Introducción}

Uno de los enfoques en la aplicación de las Tecnologías de la Información y la Comunicación (TIC) en educación, ampliamente aceptado, se basa en un nuevo paradigma de diseño de actividades de aprendizaje, poniendo énfasis en la reutilización de contenidos. Como explica Sicilia [4], al principio de la década de los 90 se popularizó la divulgación de contenidos educativos a través de Internet. Desde proyecto PROICO №30212 se investiga, modifica y desarrolla herramientas de software libre que permitan crear materiales educativos digitales. Con esto se busca favorecer un marco de colaboración para el desarrollo de las actividades académicas en los diferentes ámbitos educativos, con el fin de potenciar la generación y reusabilidad de contenidos digitales.

De lo antes dicho, surge la necesidad de desarrollar un repositorio on line para proveer el acceso a estos MED. Los repositorios se convirtieron en uno de los pilares del e-learning, hasta lograr la proclamación de la ley 26899 Repositorios digitales institucionales de acceso abierto. "Básicamente se pueden entender como: almacenes digitales en los que se recogen aportaciones individuales de los miembros de una comunidad argentina para ser compartidos y evaluados entre todos ellos."

\subsection{Recursos Educativos Abiertos}

En términos sencillos, se puede pensar que un Recurso Educativo Abierto (REA) es un material educativo, en cualquier formato (texto, imagen, audio, video, etc.) que ha sido desarrollado utilizando herramientas de software de uso libre y que su autor publica de forma abierta, es decir brindando las libertades de utilización, modificación y libre distribución; pero la definición formal abarca más que esto, la Fundación Hewlett define los REA como "recursos destinados a la enseñanza, el aprendizaje y la investigación de dominio público o que han sido liberados bajo un esquema de licenciamiento que protege la propiedad intelectual y permite su uso de forma pública y gratuita o permite la generación de obras derivadas por otros. Los Recursos Educativos Abiertos se identifican como cursos completos, materiales de cursos, módulos, libros, video, exámenes, software y cualquier otra herramienta, materiales o técnicas empleadas para dar soporte al acceso de conocimiento. (Atkins et al., 2007, p. 4)"

Desde el año 2002 la UNESCO se convirtió en la organización anfitriona de la discusión internacional en torno a esta iniciativa, cuando en el "Foro sobre Impacto de los Cursos Abiertos para Educación Superior en los países en desarrollo" se adoptó la sigla OER (del inglés Open Educational Resouces) y cuya traducción al español fue REA (Recursos Educativos Abiertos). Para ello, la UNESCO con la generosa contribución de la Fundación Flora y William Hewlett, mantiene un foro internacional de discusión con el fin de servir como un laboratorio de ideas, una central de recolección e intercambio de información, un impulsor de estándares y un catalizador de la cooperación internacional.

Teniendo como referencia la Declaración de la UNESCO en el Congreso Mundial sobre Recursos Educativos Abiertos (REA) celebrada en París en 2012, se ha decidido 
impulsar la promoción, producción, distribución y uso en Iberoamérica de licencias Creative Commons y REA. Para ello, entre otras acciones, elaborará una propuesta de licencia Creative Commons, específica para su uso en toda Iberoamérica, para la producción y uso de Recursos Educativos Abiertos.

Con respecto a esto, en Argentina se sancionó el proyecto de ley "Creación de Repositorios Digitales Abiertos de Ciencia y Tecnología". El Sistema Nacional de Repositorios Digitales (SNRD) es una iniciativa del Ministerio de Ciencia, Tecnología e Innovación Productiva conjuntamente con el Consejo Interinstitucional de Ciencia y Tecnología (CICyT) a través de sus representantes en el Consejo Asesor de la Biblioteca Electrónica de Ciencia y Tecnología.

El SNRD tiene como propósito conformar una red interoperable de repositorios digitales en ciencia y tecnología, a partir del establecimiento de políticas, estándares y protocolos comunes a todos los integrantes del Sistema. A través de su sitio web es posible acceder a las publicaciones científico-técnicas depositadas en los Repositorios argentinos adheridos al Sistema Nacional de Repositorios Digitales.

Los objetos digitales disponibles, pueden ser accedidos en forma gratuita, leídos, descargados, copiados, distribuidos, impresos, buscados o enlazados y utilizados con propósitos legítimos ligados a la investigación científica, a la educación o a la gestión de políticas públicas, sin otras barreras económicas, legales o técnicas que las que suponga Internet en sí misma. La única condición, para la reproducción y distribución de las obras, es la obligación de otorgar a los autores el control sobre la integridad de su trabajo y el derecho a ser adecuadamente reconocidos y citados.

Los MED desarrollado por nosotros cumplen los requisitos de ser recursos destinados para la enseñanza, el aprendizaje y la investigación, están liberados bajo un esquema de licenciamiento que protege la propiedad intelectual y permite su uso de forma pública, gratuita y permite la generación de obras derivadas por otros. Por lo tanto, los MED se transforman en REA y el repositorio es lo que alojará finalmente.

El presente trabajo está organizado de la siguiente manera: comienza con una introducción al tema, seguida de la conceptualización de software libre. Continúa con repositorios de código libre. Luego, se detalla el avance en el modelo de repositorio que desarrolla nuestro proyecto. Finalizamos este documento con la conclusión del mismo.

\section{2. ¿Porque elegir software libre?}

El software libre proporciona la independencia a los usuarios para ejecutar, copiar, distribuir, estudiar, cambiar y mejorar el software. Dentro de la corriente de software denominada "Software Libre" se encuentran varias aplicaciones para instalar un repositorio.

Nuestro proyecto de investigación se encuadra dentro de las premisas del software libre como lo expresa en su libro digital Richard M. Stallman:

El adjetivo «libre» en el software libre hace referencia a la libertad del usuario para ejecutar, modificar y redistribuir software. Es por ello que creemos que los usuarios de 
ordenadores deberían tener libertad para cambiar y redistribuir el software que utilizan. Esto contribuye al saber humano, al contrario que el software propietario. Por este motivo, desde esta investigación se pretende fomentar el software libre. Tal como lo promueve la ley 26.899 que fomenta a científicos y académicos a publicar sus obras. [1]

El mismo autor aporta una definición, difundida ampliamente en Internet, que aclara cual es la libertad que tienen los usuarios para ejecutar, copiar, distribuir, estudiar, cambiar y mejorar el software. Allí también, menciona las cuatro libertades para los usuarios del software:

De acuerdo con tal definición, el software es "libre" si garantiza las siguientes libertades:

Libertad 0: ejecutar el programa con cualquier propósito

Libertad 1: estudiar y modificar el programa

Libertad 2: copiar el programa de manera que se pueda ayudar al vecino o a cualquiera.

Libertad 3: mejorar el programa, y hacer públicas las mejoras.

Como se puede ver, las libertades 1 y 3 obligan a que se tenga acceso al código fuente. La libertad 2 hace referencia a la libertad de modificar y redistribuir el software libremente licenciado bajo algún tipo de licencia de software libre que beneficie a la comunidad.

Hay que aclarar que un determinado programa sea de Software libre no implica en ningún momento que este sea o deba ser gratuito (freeware). Es perfectamente compatible el que se trate de un software libre y se cobre por servicios brindados. En cuanto a la $2^{\mathrm{a}}$ premisa (la libertad de distribuir copias, con lo que puede ayudar a otros), está siempre está supeditada a los acuerdos de licencia de dicho programa (aunque se trate de programas en régimen de freeware).

Para las instituciones educativas públicas es indiscutible la conveniencia de trabajar con material de estas características.

\section{Repositorios de código abierto}

Las motivaciones que llevan el uso y desarrollo del software de código abierto son diversas, van desde razones éticas y filosóficas a cuestiones prácticas. A continuación se detalla algunas de las ventajas prácticas.

Una de las principales ventajas de los modelos de código abierto es el hecho de que el software de código abierto está disponible gratuitamente a un bajo costo, si bien esta característica no es exclusiva de software de fuente abierta, y varios productos de software propietario están a disposición en forma similar. Lo que realmente distingue el software de código abierto del software disponible sin costo es la combinación de los efectos que causan las características, tales como: el acceso al código fuente, el derecho a modificar el mismo, entre otras. Todas ellas en conjunto producen un efecto sinérgico que es la causa de las verdaderas ventajas del modelo de código abierto.

La disponibilidad del código fuente y el derecho a modificar permite el ajuste ilimitado y la mejora de un producto de software. También hace posible la adaptación del código a un nuevo hardware, para adaptarlo a las condiciones cambiantes, y para llegar a una 
comprensión detallada de cómo funciona el sistema. Esta es la razón por la que muchos expertos están llegando a la conclusión de que en realidad para extender la vida útil de una aplicación, debe estar disponible el código fuente. La disponibilidad del código fuente hace que sea mucho más fácil de aislar errores y solucionarlos.

El número de comunidades virtuales que han surgido relativas al desarrollo de repositorios y REA demuestran un gran interés en el ámbito académico. Los siguientes proyectos se encuentran trabajando con la perspectiva del Software libre: Aloha II[10], Pool[11], Eduplone[12], eRib[6], Planet[8], DOOR[5]. Entre los repositorios desarrollados de primera generación se encontró Careo [12], uno de los más conocidos que estuvo disponible de este modo.

Luego de la evaluación realizada, por el proyecto de investigación, sobre Repositorios existentes de código abierto [15] se procuró seleccionar uno que se adecuara a nuestras necesidades, por tal motivo, se optó por DOOR. La razón fundamental de esta selección es que está desarrollado en PHP y MySql, compatible con la plataforma utilizada en el proyecto para sus producciones. Además, consideramos importante que DOOR está desarrollado siguiendo el estándar internacional IMS Metadata 1.2.1 y Content Package 1.1.3. En base a las modificaciones realizadas sobre el repositorio DOOR, se dio origen al Repositorio de Objetos de Informática ROI. En este último se alojarán todos los Recurso Educativos Abiertos.

\section{Modificaciones al Repositorio}

Readecuar el repositorio DOOR para que aloje los REA, tiene como objeto que puedan ser accedidos en forma gratuita, buscados, visualizados, descargados, copiados, distribuidos, impresos, o enlazados y utilizados. Con propósitos ligados a la investigación científica, a la educación, sin barreras económicas y legales. La única condición, para la reproducción y distribución de las obras, es la obligación de otorgar a los autores el control sobre la integridad de su trabajo y el derecho a ser adecuadamente reconocidos y citados.

A continuación se detallan los cambios que realizamos al repositorio, como así también la optimización de los servicios que ofrece (buscar, cargar, descargar, etc. recursos educativos abiertos). En DOOR se pude acceder con diferentes roles, tales como: invitado (lector), administrador o usuario a cargar un REA (autor). Las modificaciones son:

1) Se llevaron a cabo modificaciones visuales para readecuarla al proyecto de investigación al cual pertenece.

2) En DOOR es posible cargar un recurso (un solo archivo a la vez), se realizaron las modificaciones necesarias para poder agregar un REA al repositorio. Por lo que los recursos de aprendizaje se pueden agregar en forma de paquetes que incluyen la descripción del objeto y el contenido o bien incluir solamente los metadatos junto con la url del objeto.

3) Se generó un árbol con categorías, para que los REA que van siendo incorporados al repositorio estén organizador a través de ellas. Esta categorización 
puede ser modificada por aquel usuario que posea el rol de administrador. Se observa en la figura 1.

4) A cada nuevo recurso que se incorpore al repositorio, se le podrá agregar el tipo de licencia "creative common" que el autor requiera. Además, cuáles serán los destinatarios del recurso, según el autor. Se realizaron modificaciones en la base de datos para que se visualice el tipo de licencia.

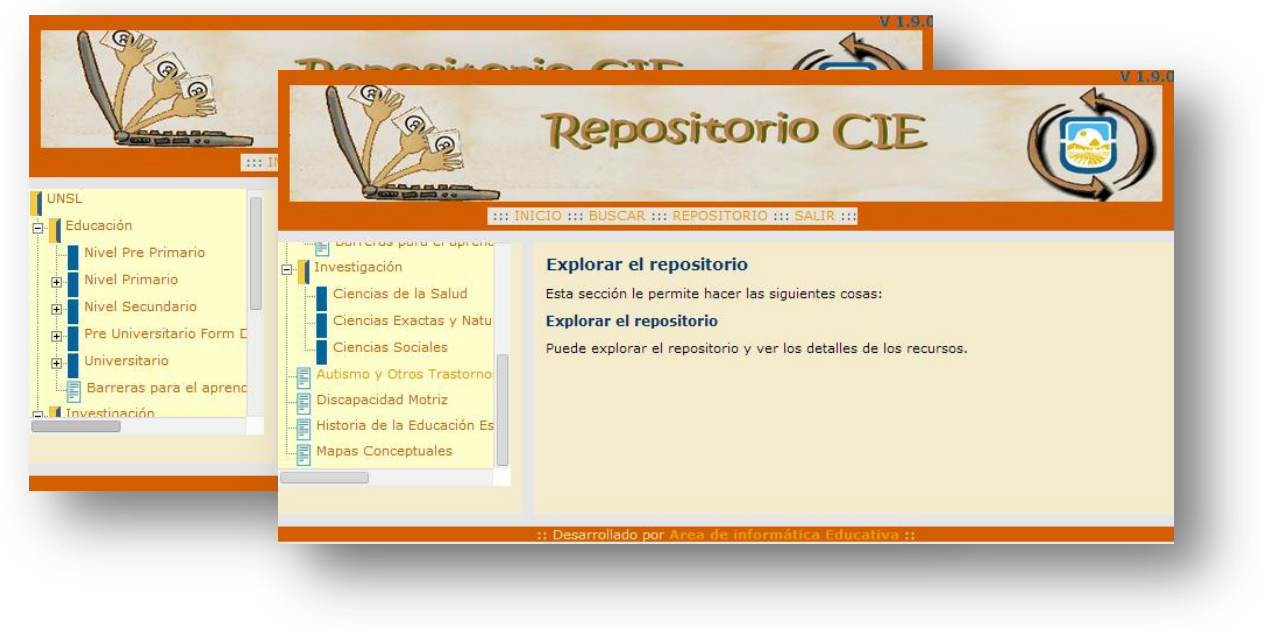

Figura 1: Visualización de categorías

En la pantalla principal del repositorio se puede observar que el acceso se realiza indicando usuario y contraseña (figura 2). Si el usuario no se ha registrado puede solicitar al administrador una cuenta para acceder. Los tipos de usuarios que se pueden asignar son los siguientes: lector, autor y administrador. Además, se generó un nuevo usuario denominado "invitado" el cual puede acceder sin que sea necesario ingresar clave y usuario.

En la figura 2 se observa que el repositorio actualmente se encuentra on-line, alojado en el servidor del proyecto. Está a disposición de docentes e investigadores que quieran alojar sus REA. 


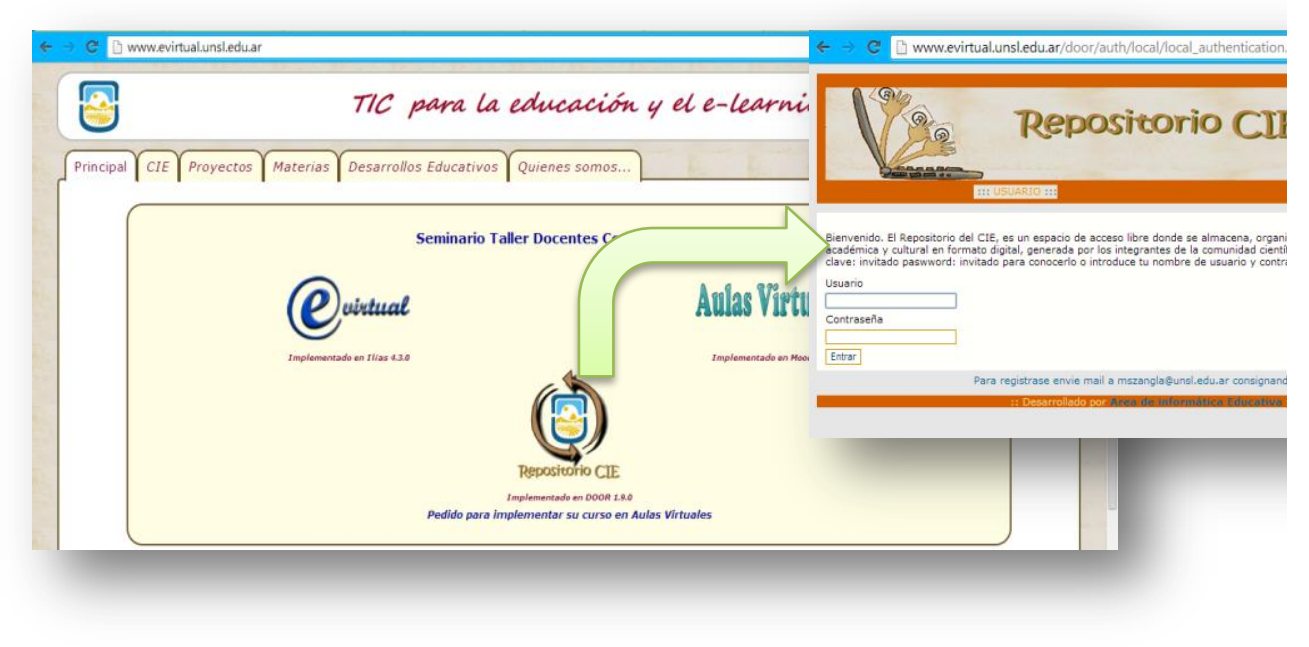

Figura 2: Servidor principal que contiene on line el Repositorio CIE

Evidentemente la creciente utilización de repositorios educativos nos lleva a observar, que en los últimos años la práctica docente ha experimentado un vertiginoso progreso en el uso de los recursos de apoyo. Es así como se ha podido observar una inclusión de herramientas sustentadas en las TIC. En consecuencia, en la actualidad podemos observar que en muchas instituciones educativas del material impreso comienzan abordar al material digitalizado, de la consulta en libros a la navegación en Internet, entre otros. Los recursos educativos, producto del avance tecnológico, están ahora al alcance de los docentes y de los alumnos. La utilización de estos recursos en el aula constituye una herramienta fundamental para el desarrollo y enriquecimiento del proceso de enseñanza-aprendizaje del alumno. A partir de estas últimas fortalezas de la inclusión de recursos es de vital importancia que toda la comunidad educativa pueda acceder a los recursos educativos abiertos, a través del repositorio, que son construidos interdisciplinariamente entre docentes de diferentes instituciones educativas.

\section{Conclusiones $\mathbf{y}$ tareas futuras}

Todo el trabajo que se viene realizando permitió que actualmente se encuentre on line la primera versión del repositorio, a cual se accede solicitando un usuario y clave. A futuro, una vez que los usuarios estén más concientizados en el uso de estos repositorios será de acceso abierto para que los usuarios puedan darse de alta. El rol seguirá siendo asignado por el administrador.

Además, se está enfocando las modificaciones del repositorio en la adecuación de la 
meta etiquetado de los REA al estándar Dublin Core [17], debido a que los repositorios más utilizados a nivel mundial utilizan estos parámetros de clasificación.

Otra propuesta para avanzar, es lograr la evaluación de pares de trabajos alojados en el repositorio, esto daría jerarquía a los mismos. Para ello es necesario que existan evaluadores que trabajaran en las distintas categorías que tienen el árbol de clasificación y un coordinador que confirmara las evaluaciones.

Nuestro equipo, seguirá trabajando en busca de compartir experiencias y conocimientos relacionados con nuestro tema central de investigación para posibilitar espacios de conocimiento colectivos.

\section{Referencias}

1. Richard M. Stallman. El Movimiento Del Software Libre. http://www.fsf.org

2. Chiarani M., Leguizamon G., Pianucci I. (2006). Repositorio de Objetos de Aprendizaje para Carreras Informáticas. WICC 2006, Moron.

3. Software libre. Free software foundation. http://www.fsf.org/

4. Sicilia Urbán Miguel-Angel, Sánchez Alonso Salvador. (2006) Repositorios de objetos de aprendizaje. Information Engineering Research Unit. Universidad de Alcalá.

5. DOOR. http://door.elearninglab.org/website/index_ita.php

6. eRIB. Repositorio Edusource. http://edusource.licef.teluq.uquebec.ca/ese/fr/install_erib.htm

7. IEEE Learning Technology Standards Committee (2002) Learning Object Metadata (LOM), IEEE 1484.12.1-2002. http://www.um.es/ead/red/M2/

8. Planet. http://ants.etse.urv.es/planetdr

9. Repositorio institucional E-Print. http://www.eprints.org/

10. Aloha II. http://aloha.netera.ca

11. Pool. Portal para Edusplash. http://edusplash.net

12. Eduplone. http://www.eduplone.net

13. Repositorio digital DSpace. http://www.dspace.org/

14. Ponce Viviana, Chiarani Marcela, Pianucci Irma. ROI: Repositorios de Objetos de Aprendizaje Informáticos. CACIC 2007. Corrientes. ISBN 950-656-109-3.

15. Chiarani M., Pianucci I., Leguizamon G. (2006). Repositorio de Objetos de Aprendizaje para Carreras Informáticas. Publicado en el WICC. Morón Disponible en CD

16. Dublin Core. http://www.dublincore.org/projects/

17. Atkins D. , Brown J. , Hammond A. (2007). A Review of the Open Educational Resources (OER)

Movement: Achievements, Challenges, and New Opportunities consultado el 6 de junio de 2013, disponible en:

http://www.hewlett.org/uploads/files/ReviewoftheOERMovement.pdf 